\title{
Las prácticas pedagógicas en la formación inicial de profesores. Análisis desde la perspectiva de la construcción del conocimiento profesional del profesor de ciencias
}

Rojas Quitian Martha'

Categoría 2. Trabajo de investigación

\section{Resumen}

Se presenta la investigación en curso en torno a la relación que puede establecerse entre la práctica pedagógica que realizan los profesores de ciencias en formación inicial y la construcción del conocimiento profesional, en tres programas de Licenciatura de la Universidad Pedagógica Nacional. Desde esta perspectiva, se propone la realización de una evaluación de la percepción de los responsables académico-administrativos, profesores en formación inicial que desarrollan práctica pedagógica, así como egresados de los programas académicos, para establecer si existe un aporte de aproximación y/o desarrollo de quince aspectos del conocimiento profesional de los profesores de ciencias.

\section{Palabras Clave}

Formación inicial de profesores, Práctica pedagógica, Conocimiento profesional

\section{Objetivo}

Caracterizar la contribución de la práctica pedagógica a la construcción del conocimiento profesional del profesor de ciencias mediante la indagación de la percepción de responsables académicos-administrativos, profesores de ciencias en formación inicial y continuada de los programas de Licenciatura en Biología, Licenciatura en Física y Licenciatura en Química de la Universidad Pedagógica Nacional.

1 Departamento de Química, Universidad Pedagógica Nacional. rojasmartha@gmail.com 
Revista Tecné, Episteme y Didaxis: TED. Año 2014, Número Extraordinario. ISSN Impreso: 0121-3814, ISSN web: 2323-0126

Memorias, Sexto Congreso Internacional sobre Formación de Profesores de Ciencias. 08 al 10 de octubre de 2014, Bogotá

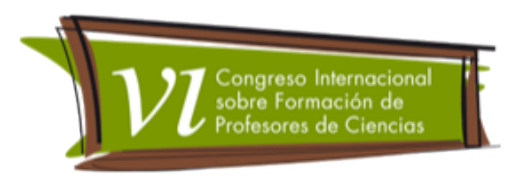

\section{Marco teórico}

El trabajo de investigación tiene dos referentes fundamentales: por una parte, los trabajos desarrollados en torno al conocimiento profesional de los profesores, y por otra, aquellos desarrollados en el campo de la práctica pedagógica. En la primera línea, las investigaciones se remontan a los estudios acerca del pensamiento del profesor. Zamudio Franco (2003) ha realizado una panorámica de las principales preocupaciones y tendencias teóricas en esos estudios iniciales:

- Teoría de los constructos. Considera el pensamiento como un proceso que configura constructo (s) diferenciales, según los estilos de enseñanza. Kelly define el constructo como la síntesis mental que cada sujeto utiliza como espacio interpretativo de su propio mundo.

- Teoría Cognitiva. Considera que la investigación sobre el pensamiento del profesor evidencia la preocupación que existe por conocer: que el profesor es un profesional racional, reflexivo, que toma decisiones, emite juicios, tiene creencias y forma rutinas profesionales.

- Interaccionismo simbólico. Se propone la búsqueda del sentido representacional y atribución de contextos (la escuela, el aula), espacios de trabajo relacional, ha evidenciado la importancia del discurso del profesor en el aula y la relación que existe entre el pensamiento del profesor y el discurso que explicita en el aula.

- La Biografía. Concede gran importancia al conocimiento de la línea de vida del profesor, a las claves de acción de su práctica y a la identificación de referencias significativas en la formación del profesor.

- Teoría de las Decisiones. La competencia por excelencia de los profesores es prepararlos para que en las complejas decisiones del aula elijan la competencia más adecuada a los sucesos que tienen lugar en cada espacio (tiempo de la enseñanza).

Con posterioridad, y a propósito del concepto de conocimiento pedagógico del contenido (PCK) propuesto por Shulman (2005), se iniciaron las investigaciones relacionadas con el conocimiento profesional de los profesores. Durán Camelo (2012) sintetiza los principales aspectos relacionados en la categorización del conocimiento profesional de los profesores: 
Revista Tecné, Episteme y Didaxis: TED. Año 2014, Número Extraordinario. ISSN Impreso: 0121-3814, ISSN web: 2323-0126

Memorias, Sexto Congreso Internacional sobre Formación de Profesores de Ciencias. 08 al 10 de octubre de 2014, Bogotá

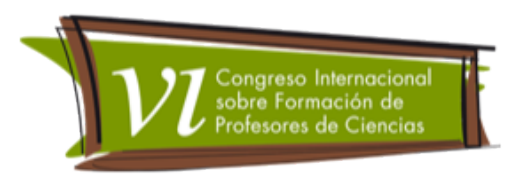

Tabla 1. Componentes del conocimiento profesional de los profesores (Durán Camelo, 2012)

\begin{tabular}{|c|c|}
\hline Shulman (1986) & $\begin{array}{l}\text { Conocimiento de la materia } \\
\text { Conocimiento pedagógico general } \\
\text { Conocimiento didáctico del contenido } \\
\text { Conocimiento de los objetivos de la enseñanza } \\
\text { Conocimiento curricular }\end{array}$ \\
\hline $\begin{array}{c}\text { Grosman } \\
\text { (1990) }\end{array}$ & $\begin{array}{l}\text { Conocimiento de la materia } \\
\text { Conocimiento pedagógico general } \\
\text { Conocimiento didáctico del contenido } \\
\text { Conocimiento de los propósitos de enseñanza } \\
\text { Conocimiento de estrategias de enseñanza } \\
\text { Conocimiento del contexto } \\
\text { Conocimiento curricular }\end{array}$ \\
\hline $\begin{array}{c}\text { Martín del Pozo } \\
\text { (1994) }\end{array}$ & $\begin{array}{l}\text { Conocimiento profesionalizado del contenido } \\
\text { Conocimiento pedagógico general } \\
\text { Conocimiento didáctico Específico } \\
\text { Conocimiento Práctico }\end{array}$ \\
\hline $\begin{array}{l}\text { Azcárate } \\
\text { (1995) }\end{array}$ & $\begin{array}{l}\text { Conocimiento profesionalizado de la disciplina } \\
\text { Conocimiento práctico profesional } \\
\text { Conocimiento experiencial }\end{array}$ \\
\hline
\end{tabular}

En el área específica del conocimiento profesional del profesor de ciencias, se destacan los aportes de Barnet y Hodson (2001) quienes proponen que dicho conocimiento está dado, entre otros, por el conocimiento del contexto de enseñanza que está determinado por (1) el conocimiento sobre la enseñanza de las Ciencias (metas que persigue la comunidad académica de la enseñanza de las Ciencias, y la educación ambiental), (2) el profesionalismo del profesor (competencias básicas docentes, interrelaciones entre profesores y credibilidad entre colegas), (3) el currículo de Ciencias (los contenidos y evaluaciones prescritos), y (4) el conocimiento de la cultura específica escolar (patrones de conducta aceptables en lo local).

Por otra parte, Gess-Newsome y Lederman (1999) establecen que el conocimiento profesional del profesor de ciencias está signado, además, por un 
Revista Tecné, Episteme y Didaxis: TED. Año 2014, Número Extraordinario. ISSN Impreso: 0121-3814, ISSN web: 2323-0126

Memorias, Sexto Congreso Internacional sobre Formación de Profesores de Ciencias. 08 al 10 de octubre de 2014, Bogotá

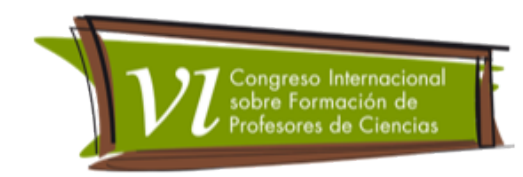

conocimiento del contenido disciplinar definido a partir de la comprensión de (1) los contenidos disciplinares (factores, conceptos, principios y procedimientos propios de la disciplina), (2) la estructura disciplinar (interrelación entre los conceptos - "secuenciación"), (3) la naturaleza disciplinar (NOS), (4) las orientaciones para la enseñanza de contenidos específicos, así como (5) la influencia contextual en la implementación escolar.

Finalmente, Valbuena (2007) propone que el conocimiento profesional de los profesores de ciencias atiende a un conocimiento pedagógico (Proceso de enseñanza-aprendizaje - sus características, los factores que inciden, los principales obstáculos, las estrategias para facilitarlo) como también a un conocimiento didáctico del contenido objeto de enseñanza (los contenidos [disciplinares] por enseñar, las finalidades de la [disciplina] y de su enseñanza, las estrategias metodológicas para la enseñanza de la disciplina, las concepciones de los alumnos acerca de la disciplina, las dificultades en la enseñanza aprendizaje de la disciplina y La evaluación de los aprendizajes de la disciplina.

Ahora bien, en lo referente a la práctica pedagógica como objeto de investigación existe una tradición de más de dos décadas acerca de los propósitos a los que esta práctica debe apuntar y entre los que se cuentan:

- La oferta de un acercamiento a la docencia real; la posibilidad del ensayo de instrumentos y métodos; el suministro de una base experimental para hacer más significativos los conocimientos teóricos; la ayuda a la innovación y mejora de la realidad escolar (Gimeno y Fernández, 1980).

- La comprobación del interés del profesor en formación por su profesión; el acercamiento al conocimiento real de los alumnos (intereses, necesidades, diferencias individuales, etc.); el conocimiento del funcionamiento de un centro escolar y de una clase; la comprensión de la escuela como una empresa colectiva fruto de las relaciones positivas de sus miembros; la resolución de las rutinas ordinarias: ordenar, clasificar el material, aplicar técnicas de disciplina; el aprendizaje de profesores con mayor experiencia; la comprobación de la adecuación de los conocimientos académicos a la realidad escolar (Benejam, 1993).

- Analizar el centro como medio educativo institucional y los elementos implicados en la interrelación centro educativo-medio social; Identificar y 
Revista Tecné, Episteme y Didaxis: TED. Año 2014, Número Extraordinario. ISSN Impreso: 0121-3814, ISSN web: 2323-0126

Memorias, Sexto Congreso Internacional sobre Formación de Profesores de Ciencias. 08 al 10 de octubre de 2014, Bogotá

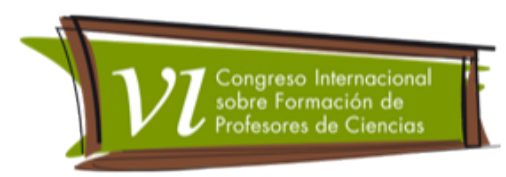

analizar los aspectos psicopedagógicos implicados en la enseñanza: técnicas de observación, empleo de técnicas y recursos, vinculación teórico - práctica, etc. Desarrollar actitudes y valores que vayan configurando un estilo en el futuro profesor (Maña y Villanueva, 1987).

- La facilitación de la traslación de conocimientos aprendidos a situaciones reales de aula; el aprendizaje desde la práctica de aspectos no estudiados teóricamente; la construcción de conocimiento pedagógico a través de la práctica (Montero, 1990).

- El contacto con una situación real y el conocimiento, de manera sistemática, de su funcionamiento general y la práctica que en ella se realiza; la iniciación en el trabajo y las destrezas profesionales específicas; la adquisición de aprendizajes relacionados con la cultura profesional, así como de cierta conciencia sobre sí mismo; la reflexión sobre la actividad desarrollada y sobre las experiencias de las prácticas en su conjunto (Zabalza, 1990).

Zeichner (1983) ha propuesto que la práctica pedagógica podría clasificarse según el modelo al que atiende y los objetivos que persiga, en tres grupos:

- Práctica docente. Las prácticas son esenciales para adquirir las técnicas del oficio de ser maestro. El esquema tradicional para su desarrollo consiste en: información - observación - imitación de profesores experimentados. Se observa una clara separación entre la teoría y la práctica.

- Práctica social. Las prácticas son el espacio para contribuir al desarrollo integral del futuro profesor pues le permite acercarse de lleno a la realidad de las instituciones educativas e incidir directamente en ellas. El enfoque de práctica se corresponde con los proyectos sociales comunitarios en cuyo trasfondo subyace la idea de cumplir una misión con las comunidades deprimidas. El practicante se entrega de lleno a contribuir a la solución de problemas de la comunidad.

- Práctica investigativa. La práctica proporciona capacidad de análisis de la acción, de las creencias y teorías implícitas que subyacen en ellas, de los significados otorgados por los protagonistas de la acción y del bagaje que los futuros profesores traen ya a la formación. El enfoque de práctica considera necesario integrar la teoría y la práctica pues supone que la práctica es un espacio para lograr conocimientos nuevos, que deben analizarse a profundidad. 
Revista Tecné, Episteme y Didaxis: TED. Año 2014, Número Extraordinario. ISSN Impreso: 0121-3814, ISSN web: 2323-0126

Memorias, Sexto Congreso Internacional sobre Formación de Profesores de Ciencias. 08 al 10 de octubre de 2014, Bogotá

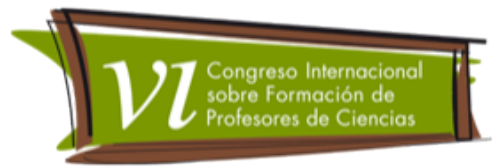

\section{Metodología}

A partir de estos presupuestos teóricos se ha planteado la realización de un análisis exploratorio de percepción por categorización deductiva a los responsables académicos y administrativos de prácticas pedagógicas, los directores, coordinadores y asesores de prácticas pedagógicas vinculados a los programas de licenciatura de interés. Las categorías que son base para la evaluación son quince (15) componentes del conocimiento profesional del profesor de ciencias (Gess-Newsome y Lederman, 1999, Barnet y Hodson, 2001, Valbuena, 2007), con el ánimo de interrogar sobre la contribución que para cada uno puede tener la práctica pedagógica. Dicha contribución se propone desde dos valoraciones:

- Aproximación, si desde la percepción del responsable académico solo hasta que se alcanza la instancia de la práctica pedagógica los estudiantes se enfrentan a la reflexión y trabajo acerca de cada componente. El criterio que sustenta esta valoración es que al final de la práctica pedagógica, los profesores en formación inicial demuestran una comprensión preliminar del componente pues están en la capacidad de enunciarlo y definirlo (al menos parcialmente).

- Desarrollo, si desde la percepción del responsable académico cuando se alcanza la instancia de la práctica pedagógica los estudiantes continúan con la reflexión y trabajo acerca de cada componente en articulación con los espacios académicos del programa de formación. El criterio que sustenta esta valoración es que al final de la práctica pedagógica, los profesores en formación inicial demuestran una comprensión profunda del componente pues están en la capacidad de describirlo (detalladamente) y explicar su papel e importancia.

\section{Referencias bibliográficas}

Barnett, J., y Hodson, D. (2001). Pedagogical context knowledge: Toward a fuller understanding of what good science, teachers know. Science Education, 85(4), 426-453.

Benejam, P. (1993). Los contenidos de la Didáctica de las Ciencias Sociales en la formación del profesorado. Las Didácticas específicas en la formación 
Revista Tecné, Episteme y Didaxis: TED. Año 2014, Número Extraordinario. ISSN Impreso: 0121-3814, ISSN web: 2323-0126

Memorias, Sexto Congreso Internacional sobre Formación de Profesores de Ciencias. 08 al 10 de octubre de 2014, Bogotá

del profesorado (págs. 341-347). Santiago de Compostela: Tórculo.

Durán Camelo, V. H. (2012). Antecedentes y perspectivas investigativas del conocimiento profesional docente en actividad fisica en el ámbito comunitario. Lúdica Pedagógica, 2(17), 153-163.

Gess-Newsome, J., y Lederman, N. G. (Eds.). (1999). examining pedagogical content knowledge: the construct and its implications for science education. Contemporary Trends and Issues in Science Education. Dordrecht; Boston: Kluwer Academic.

Gimeno, J., y Fernández, M. (1980). La formación del profesorado de EGB. Análisis de la situación española. Madrid: MEC.

Maña, T., y Villanueva, M. (1987). Las Prácticas en la escuela de maestros. San Cugat: Universidad Autónoma de Barcelona.

Montero, L. (1990). Modelos de prácticas: modelo implícito de prácticas que revelan los actuales programas de formación inicial del profesorado. La formación práctica de los profesores (págs. 41-52). Presentado en Simposio sobre prácticas escolares, Santiago: Actos.

Shulman, L. S. (2005). Conocimiento y enseñanza: fundamentos de la nueva reforma. Profesorado: Revista de curriculum y formación del profesorado, $9(2), 1$.

Valbuena Ussa, E. O. (2007). El conocimiento didáctico del contenido biológico: Estudio de las concepciones disciplinares y didácticas de futuros docentes de la Universidad Pedagógica Nacional (Colombia) (Tesis Doctoral). Madrid: Universidad Complutense de Madrid.

Zabalza, M. A. (1990). Teoría de las prácticas. La formación práctica de los profesores. La formación práctica de los profesores (págs. 25-27). Presentado en Simposio sobre prácticas escolares, Santiago: Actos.

Zamudio Franco, J. I. (2003). El conocimiento profesional del profesor de Ciencias Sociales. Revista de Teoría y Didáctica de las Ciencias Sociales, (8), 87-103.

Zeichner, K. M. (1983). Altemative paradigms of teacher education. Journal of teacher education, 34(3), 3-9. 